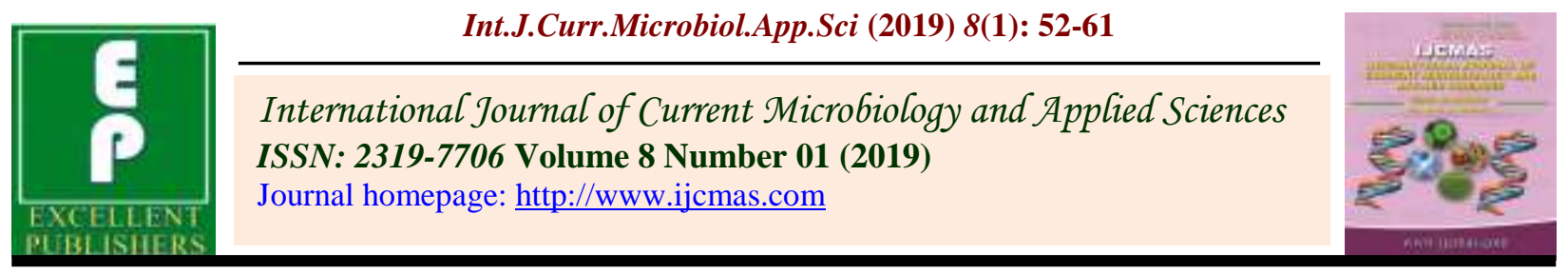

Original Research Article https://doi.org/10.20546/ijcmas.2019.801.007

\title{
Heterosis Studies for Yield and Yield Attributing Traits in Tomato (Solanum lycopersicum L.) under North Western Himalayan Region, India
}

\author{
Shilpi Khar* and Divya Arti \\ Department of Vegetable Science, Dr. Yashwant Singh Parmar University of Horticulture \& \\ Forestry, Nauni, Solan, HP Pin code 173230, India \\ *Corresponding author
}

\section{A B S T R A C T}

\section{Keywords}

Heterosis, Hybrids, Line $\times$ Tester,

Quantitative,

Qualitative and

Tomato

Article Info

Accepted:

12 December 2018

Available Online:

10 January 2019
Line $\mathrm{x}$ Tester analysis was performed by employing fourteen tomato genotypes (10 lines and 4 testers) to develop 40 hybrids. These hybrids along with parental lines and commercial check Naveen 2000+ were evaluated to know the extent of heterosis exhibited to different yield and yield attributing traits. Results revealed that nine hybrid combinations produced significantly increased heterotic effect over the better parent. The top best 5 such combinations were; EC-5863 x Solan Lalima, EC-5863 x Solan Vajr, CLN 2123 A-1 x Solan Lalima, EC-526146 x Solan Lalima and CLN 2123 A-1 x Solan Vajr which registered superiority in term of yield/plant and yield/hectare to the tune of 68.18 , $68.00,67.15,64.87$ and 48.28 per cent, respectively over the better parent. Whereas, 6 cross combinations, reported significant positive heterosis over the standard check viz. EC5863 x Solan Lalima (26.24 \%) followed by EC-5863 x Solan Vajr (25.73 \%), CLN 2123 A-1 x Solan Lalima (25.46 \%), EC-526146 x Solan Lalima (23.75 \%), CLN 2123 A-1 x Solan Vajr (48.23 \%) and EC-521041 x Solan Lalima (1.93 \%) these hybrids also reported heterosis for yield attributing traits.

\section{Introduction}

Tomato (Solanum lycopersicum L.) being the crop of importance for both culinary and processing purpose, it has been cultivated over large area around the world. This crop exhibits rich genetic diversity for various horticultural traits and has a scope for its improvement. In tomato, the comparative ease of emasculation, high percentage of fruit setting and good number of seeds per fruit also facilitate the exploitation of heterosis. Yield being a complex quantitative character in tomato can be improved through improving its contributing traits, i.e. mean fruit weight, number of fruits per plant, fruit length, and breadth and plant vigour. The genetic improvement of crop plants and exploitation of heterosis requires the selection of suitable parents and cross combinations. The selection of parents on the basis of per se performance does not necessarily lead to desirable results (Allard, 1960). Heterosis for various fruit quantitative and quality characters has been reported by Sahu et al., (2016) and Panchal et al., (2017) in tomato Hence, present 
investigation was carried out at YS Parmar University of Horticulture and Forestry, Nauni, Solan during 2016 and 2017 to assess the heterosis levels expressed by hybrids over their parents for Yields and yield attributing traits taken under consideration and also to identify best heterotic combinations for the same.

\section{Materials and Methods}

Source materials for current study comprised of fourteen diverse genotypes. Six lines (CLN 2070 B-1, CLN 2116 B-1, CLN 2123 A-1, BWR-1, BWR-5, EC- 528372, EC- 521041, EC- 526146, EC- 5863, EC- 521079) and four testers (FT-5, Solan Lalima, Solan Vajr and Arka Meghali) were crossed in Line X Tester fashion at Experimental Farm Department of Vegetable YS Parmar UHF, Nauni, Solan during Rabi of 2016. Evaluation of hybrids for various Yield and yield attributing characters in comparison to their parents along with a standard check (Naveen 2000+) was taken up during summer 2017 in randomized block design with three replications. About 5 fruits from each replication of an entry were considered to record observation for the traits plant height, days to first flowering, days to first harvest, fruit shape index, fruit firmness, pericarp thickness, number of locules, number of fruits per cluster, number of fruits per plant, average fruit weight, marketable fruit yield per plant, yield per hectare, alternaria blight severity, buckeye rot incidence, total soluble solids, ascorbic acid content and lycopene content Heterosis values in negative direction were considered as desirable for the character days to first harvest, days to first flowering number of locules per fruit, alternaria blight severity and buckeye rot incidence.

\section{Results and Discussion}

Analysis of variance for seventeen considered yield and yield attributing traits (Table 1) revealed that mean sum of squares for parents and hybrids were significant for all the traits except days to first flowering and number of fruits per cluster indicating presence of heterosis for these characters. Significant positive heterosis for plant height was observed in 11 hybrids over the better parent (BP) as seen through Table 2. Maximum increased heterosis over better parent was found in cross combination EC-521079 $\mathrm{x}$ Solan Lalima $(64.35 \%)$. Over the standard check (SC), 3 of the combinations viz. EC521041 x Solan Lalima followed by CLN 2123 A-1 x Solan Lalima and EC-526146 x Solan Lalima gave significant increased heterosis to the tune of 7.89, 5.84 and 5.66 percent, respectively. The results obtained are in line with Fageria et al., (2001). For days to first flowering significant negative heterosis over better parent was observed in as many as 13 cross combinations, maximum being in EC-521079 x Solan Lalima (-20.87 \%) as enumerated through Table 2. Negative heterosis for this trait over the better parent has also been reported by Baishya et al., (2001) Over the standard check, only one cross combination i.e. EC-521079 x Solan Lalima $(-8.87 \quad \%)$ recorded desirable significant heterosis. Maximum significant negative heterosis over better parent (\%) was found in EC-521079 x Solan Lalima (-20.43) for days to first harvest. However, over the standard check, CLN 2123 A-1 x Solan Lalima (-14.68) showed significantly highest negative heterosis similar reports were obtained by Singh et al., (2008). For fruit shape index positive heterotic effect (\%) over better parent was reported in 6 cross combinations, highest being in EC-5863 x Solan Vajr (8.03). Over the standard check, as many as 24 crosses showed significant positive heterosis, with maximum in EC-5863 $x$ Solan Lalima (15.38). The results are in line with Premalakshme et al., (2006). Significant positive heterosis (\%) over better parent was observed in only 3 cross combinations i.e. EC- 
528372 x Arka Meghali (32.44) followed by EC-521041 x FT-5 (22.41) and CLN 2070 B-1 x FT-5 (15.11) for fruit firmness (Table 2). Over the standard check, 19 cross combinations were positively heterotic, out of which the best cross combinations was; EC5863 x Solan Vajr (60.71). Results are in accordance with Joshi et al., (2004). Significantly positive heterosis over better parent was found in 3 cross combinations namely EC-526146 x FT-5 (52.54 \%), EC528372 x Solan Lalima (20.83 \%) and BWR-5 $\mathrm{x}$ Solan Lalima $(8.45 \%)$ for pericarp thickness (Table 3).

Whereas, 4 cross combinations viz. EC-5863 x Solan Lalima (18.60 \%), EC-521041 x Solan Lalima (17.94 \%), EC-526146 x FT-5 (15.38 $\%$ ) and CLN 2123 A-1 x Solan Lalima (14.75 $\%)$ showed significantly positive heterosis over the standard check the results are in accordance with Sharma and Thakur (2007). Since less number of locules are desirable in tomato, so heterosis over better parent was found significantly negative in 10 cross combinations, maximum being in BWR-5 x Solan Lalima (-67.64 \%) (Table 3). Over the check, as many as 22 combinations revealed negative heterosis, maximum being in EC521041 x Solan Lalima (-39.72 \%). Similar results were obtained by Kurian et al., (2001). For number of fruits per cluster only one i.e., CLN 2116 B-1 x Solan Lalima showed significant positive heterosis over better parent $(7.44 \%)$ as well as standard check $(17.11 \%)$ heterosis for number of fruits per cluster was also reported by Kumar et al., (2012). Highest significant heterosis for number of fruits per plant (Table 4) over better parent was found in 3 of the hybrid combinations viz. EC-526146 x FT-5 (11.82 \%), EC-5863 x Solan Vajr $(11.32 \%)$ and EC- 5863 x Solan Lalima (10.66\%) whereas, 5 cross combinations viz. EC-5863 x Solan Lalima (30.20 \%), EC-5863 x Solan Vajr (22.50 \%), EC-521041 x Solan Lalima (22.14 \%), EC-526146 x Solan Lalima
(16.17 \%) and CLN 2123 A-1 x Solan Lalima $(14.68 \%)$ showed significant positive heterosis over the check hybrid (Naveen 2000 $+)$ results are in line with the findings of Yadav et al., (2013). Significant positive heterosis for fruit weight over better parent was found in 18 cross combinations (Table 4) with maximum heterosis in; BWR-5 x Arka Meghali (67.58 \%). Whereas, Over the standard check, 2 of the crosses; EC-526146 x FT-5 (8.64 \%) and CLN 2123 A-1 x Solan Vajr $(7.17 \%)$ showed significant positive heterosis. Results are in accordance with Kurian et al., (2001).

Nine hybrid combinations produced significantly increased heterotic effect over the better parent in term of yield/plant and yield per hectare. The top best 5 such combinations were; EC-5863 x Solan Lalima, EC-5863 x Solan Vajr, CLN 2123 A-1 x Solan Lalima, EC-526146 x Solan Lalima and CLN 2123 A-1 x Solan Vajr which registered superiority to the tune of $68.18,68.00,67.15$, 64.87 and 48.28 per cent, respectively over the better parent. Over the standard check, 6 cross combinations reported significant positive heterosis viz. EC-5863 x Solan Lalima (26.24 $\%)$ followed by EC-5863 x Solan Vajr (25.73 \%), CLN 2123 A-1 x Solan Lalima (25.46 \%), EC-526146 x Solan Lalima (23.75 \%), CLN 2123 A-1 x Solan Vajr (48.23\%) and EC521041 x Solan Lalima (1.93\%) (Table 4). Similar results were reported by Gaikwad and Cheema (2010). Negative heterosis is desirable for alternaria blight severity as such; 16 of the cross combinations observed significant negative heterosis $(\%)$ over better parent, maximum depicted in CLN 2116 B-1 x Solan Lalima (-62.17). Further, as many as 15 cross combinations showed desirable significant negative heterotic effects over the standard check; maximum being in CLN 2116 B-1 x Solan lalima (-47.26) (Table 5). Similar results on alternaria blight were reported by Rao et al., (2007). 
Table.1 Analysis of variance for combining ability for various traits in tomato

\begin{tabular}{|c|c|c|c|c|c|c|c|}
\hline \multirow{2}{*}{$\begin{array}{l}\text { Sr. } \\
\text { No. }\end{array}$} & Source of variation $\rightarrow$ & \multirow{2}{*}{$\begin{array}{l}\text { Replications } \\
2\end{array}$} & \multirow{2}{*}{$\begin{array}{l}\text { Crosses } \\
39\end{array}$} & \multirow{2}{*}{$\begin{array}{l}\text { Lines } \\
9\end{array}$} & \multirow{2}{*}{$\begin{array}{l}\text { Tester } \\
3\end{array}$} & \multirow{2}{*}{$\begin{array}{l}\text { Line } x \text { Tester } \\
27\end{array}$} & \multirow{2}{*}{$\begin{array}{l}\text { Error } \\
78\end{array}$} \\
\hline & $\underset{\downarrow}{\text { Trait }} \quad$ df $\rightarrow$ & & & & & & \\
\hline 1 & Plant height $(\mathrm{cm})$ & 9.96 & $1263.39 *$ & $3670.59 *$ & $299.44^{*}$ & $568.10 *$ & 18.30 \\
\hline 2 & Days to first flowering & 2.72 & $13.03 *$ & $19.66 *$ & 0.74 & $12.18 *$ & 4.50 \\
\hline 3 & Days to first harvest & 21.15 & $50.50 *$ & $82.85 *$ & $58.75^{*}$ & $38.80 *$ & 10.79 \\
\hline 4 & Fruit shape index & 0.00 & $0.09 *$ & $0.09 *$ & $0.16^{*}$ & $0.08 *$ & 0.00 \\
\hline 5 & Fruit firmness $\left(\mathrm{kg} / \mathrm{cm}^{2}\right)$ & $346901.69 *$ & $316407.63 *$ & $268889.39 *$ & $666823.93 *$ & $293311.89 *$ & 41121.44 \\
\hline 6 & Pericarp thickness (mm) & 0.07 & $2.10^{*}$ & $2.50 *$ & $2.84^{*}$ & $1.88^{*}$ & 0.20 \\
\hline 7 & Number of locules per fruit & 0.004 & $0.916 *$ & $1.63 *$ & $1.13^{*}$ & $0.66^{*}$ & 0.20 \\
\hline 8 & Number of fruits per cluster & 0.48 & $1.002 *$ & $2.32 *$ & 0.23 & $0.65^{*}$ & 0.24 \\
\hline 9 & Number of fruits per plant & 4.52 & $59.07 *$ & $119.009 *$ & $45.67 *$ & $40.59 *$ & 6.23 \\
\hline 10 & Average fruit weight (g) & 24.85 & $689.09 *$ & $304.82 *$ & $1695.04 *$ & $705.40 *$ & 11.09 \\
\hline 11 & Marketable fruit yield per plant (g) & 51.30 & $484,725.43^{*}$ & $698,923.77 *$ & $571,756.87 *$ & $403,655.82 *$ & 49.644 \\
\hline 12 & Marketable yield (q/ha) & 3.59 & $48,096.64 *$ & $69,350.44 *$ & $56,731.91 *$ & $40,052.56^{*}$ & 4.95 \\
\hline 13 & Alternaria blight severity (\%) & 21.59 & $358.40 *$ & $610.38 *$ & $119.91 *$ & $293.68 *$ & 15.01 \\
\hline 14 & Buckeye rot incidence (\%) & $98.32 *$ & $263.59 *$ & $695.77 *$ & $117.24 *$ & $135.79 *$ & 15.17 \\
\hline 15 & Total soluble solids ( ${ }^{\circ}$ Brix $)$ & $8.08 *$ & $0.55^{*}$ & $0.52 *$ & $0.39 *$ & $0.58 *$ & 0.12 \\
\hline 16 & Ascorbic acid content (mg/100g) & 3.55 & $19.13 *$ & $17.03 *$ & $38.41 *$ & $17.69 *$ & 5.44 \\
\hline 17 & Lycopene content (mg/100g) & 0.04 & $2.49 *$ & $3.48 *$ & $3.54 *$ & $2.05^{*}$ & 0.05 \\
\hline
\end{tabular}


Table.2 Estimation of heterosis for plant height, days to first flowering, days to first harvest and fruit shape index

\begin{tabular}{|c|c|c|c|c|c|c|c|c|c|}
\hline \multicolumn{10}{|c|}{ Percent increase or decrease over } \\
\hline \multirow[t]{2}{*}{ Sr. No } & \multirow[t]{2}{*}{ Crosses } & \multicolumn{2}{|c|}{ Plant height (cm) } & \multicolumn{2}{|c|}{ Days to first flowering } & \multicolumn{2}{|c|}{ Days to first harvest } & \multicolumn{2}{|c|}{ Fruit shape index } \\
\hline & & BP & SC & BP & $\mathrm{SC}$ & BP & $\mathrm{SC}$ & BP & $\mathrm{SC}$ \\
\hline 1 & CLN 2070 B-1 x FT-5 & $9.28^{*}$ & $-7.13^{*}$ & -1.23 & 4.55 & -3.52 & 3.16 & -0.30 & $7.39 *$ \\
\hline 2 & CLN 2116 B-1 x FT-5 & -14.13 & $-13.37^{*}$ & 4.29 & $10.39^{*}$ & -3.89 & 1.94 & $-34.97 *$ & $-27.88^{*}$ \\
\hline 3 & CLN 2123 A-1 x FT-5 & $5.51^{*}$ & $-6.10^{*}$ & -1.34 & $11.47^{*}$ & 0.92 & 7.04 & $-4.45^{*}$ & 3.19 \\
\hline 4 & BWR-1 x FT-5 & 54.25 & $-14.35^{*}$ & $-11.25^{*}$ & -6.06 & $-14.70^{*}$ & $-7.77^{*}$ & -6.83 & 0.64 \\
\hline 5 & BWR-5 x FT-5 & 2.99 & $-12.48 *$ & $-11.31^{*}$ & -1.52 & $-14.64^{*}$ & $-8.74^{*}$ & $-7.42^{*}$ & 0.00 \\
\hline 6 & EC-528372 x FT-5 & $-7.95^{*}$ & $-7.13^{*}$ & -9.36 & 0.65 & $-11.39 *$ & $-10.31 *$ & $-2.02^{*}$ & $8.65^{*}$ \\
\hline 7 & EC-521041 x FT-5 & $6.16^{*}$ & $-5.53^{*}$ & -9.96 & 1.73 & 3.00 & 4.25 & $-1.48^{*}$ & $6.16^{*}$ \\
\hline 8 & EC-526146 x FT-5 & 43.07 & $-5.70^{*}$ & 1.37 & $12.56^{*}$ & -4.94 & 2.79 & $3.56^{*}$ & $11.86^{*}$ \\
\hline 9 & EC-5863 x FT-5 & -21.13 & $-16.18^{*}$ & $8.84^{*}$ & $11.91^{*}$ & $-14.08^{*}$ & $-8.13^{*}$ & $-8.17^{*}$ & $4.62^{*}$ \\
\hline 10 & EC-521079 x FT-5 & -20.80 & $-15.82^{*}$ & 9.80 & 6.71 & 11.17 & 0.24 & -8.73 & $3.85^{*}$ \\
\hline 11 & CLN 2070 B-1 x Solan Lalima & $-31.61^{*}$ & $-27.32^{*}$ & -2.68 & $9.96^{*}$ & -0.27 & $-10.07 *$ & -4.23 & $8.97 *$ \\
\hline 12 & CLN 2116 B-1 x Solan Lalima & $-12.91 *$ & $-7.44 *$ & $-6.29^{*}$ & -6.49 & $-16.95^{*}$ & $-10.19 *$ & $-7.61^{*}$ & $5.14 *$ \\
\hline 13 & CLN 2123 A-1 x Solan Lalima & $24.54 *$ & $5.84^{*}$ & -5.07 & 1.30 & -21.80 & $-14.68^{*}$ & 7.10 & $11.22^{*}$ \\
\hline 14 & BWR-1 x Solan Lalima & $-6.14 *$ & -5.308 & 3.65 & $10.61^{*}$ & $-17.24 *$ & $-9.71^{*}$ & -2.89 & $7.47 *$ \\
\hline 15 & BWR-5 x Solan Lalima & $-17.68 *$ & $-26.74^{*}$ & $-13.79^{*}$ & -2.60 & -20.02 & $-12.74 *$ & 2.09 & $9.62^{*}$ \\
\hline 16 & EC-528372 x Solan Lalima & 38.90 & 1.52 & $1.22^{*}$ & $8.01 *$ & -5.45 & 3.16 & $-3.13^{*}$ & $-30.45^{*}$ \\
\hline 17 & EC-521041 x Solan Lalima & $26.95^{*}$ & $7.89^{*}$ & $-16.35^{*}$ & -3.68 & -16.01 & $-10.19 *$ & $6.48^{*}$ & $10.58^{*}$ \\
\hline 18 & EC-526146 x Solan Lalima & $4.73^{*}$ & $5.66^{*}$ & $-18.42^{*}$ & -6.06 & 0.97 & $-11.16^{*}$ & 2.02 & $13.14^{*}$ \\
\hline 19 & EC-5863 x Solan Lalima & $18.58^{*}$ & $-5.53^{*}$ & -12.41 & 0.87 & 1.53 & $-11.53^{*}$ & 7.46 & $15.38^{*}$ \\
\hline 20 & EC-521079 x Solan Lalima & $64.35^{*}$ & $-6.10^{*}$ & $-20.87 *$ & $-8.87 *$ & $-20.43 *$ & $-13.96 *$ & $-0.93^{*}$ & $-31.73^{*}$ \\
\hline 21 & CLN 2070 B-1 x Solan Vajr & $-16.15^{*}$ & $-28.74 *$ & $-8.00^{*}$ & -5.41 & $-9.31^{*}$ & -3.03 & 5.86 & $9.94 *$ \\
\hline 22 & CLN 2116 B-1 x Solan Vajr & $-16.12^{*}$ & $-15.37^{*}$ & 0.00 & -1.95 & -3.07 & -4.25 & $0.87^{*}$ & $11.86^{*}$ \\
\hline 23 & CLN 2123 A-1 x Solan Vajr & $4.81^{*}$ & $-6.738^{*}$ & $-13.99^{*}$ & -2.81 & -9.09 & $-10.19 *$ & 2.69 & $10.26^{*}$ \\
\hline 24 & BWR-1 x Solan Vajr & $31.86^{*}$ & $-29.55^{*}$ & $1.52^{*}$ & 1.30 & $-15.60 *$ & $-8.74^{*}$ & 31.27 & $8.97 *$ \\
\hline 25 & BWR-5 x Solan Vajr & -14.31 & $-14.08 *$ & $-7.00^{*}$ & 0.65 & -16.23 & $-10.44^{*}$ & $-38.23^{*}$ & $-28.53^{*}$ \\
\hline 26 & EC-528372 x Solan Vajr & $-24.12^{*}$ & $-23.44 *$ & $-11.40^{*}$ & -4.11 & $14.06^{*}$ & 6.31 & $-1.94 *$ & $13.46^{*}$ \\
\hline 27 & EC-521041 x Solan Vajr & $-6.58^{*}$ & $-6.33^{*}$ & -0.38 & $12.56^{*}$ & -5.73 & $-12.13^{*}$ & $-6.93^{*}$ & $7.52^{*}$ \\
\hline 28 & EC-526146 x Solan Vajr & $-12.80^{*}$ & $-12.57^{*}$ & $-11.80^{*}$ & -4.55 & -15.49 & $-8.62^{*}$ & $-29.92^{*}$ & $-18.91^{*}$ \\
\hline 29 & EC-5863 x Solan Vajr & $4.84^{*}$ & -0.49 & 1.69 & $17.31^{*}$ & $-10.44^{*}$ & -4.25 & 8.03* & $12.18^{*}$ \\
\hline 30 & EC-521079 x Solan Vajr & $-26.90^{*}$ & $-26.25^{*}$ & -8.63 & 5.41 & 20.13 & $8.62^{*}$ & -4.05 & $6.54^{*}$ \\
\hline 31 & CLN 2070 B-1 x Arka Meghali & $-36.20^{*}$ & $-39.44 *$ & -14.63 & -1.52 & 17.85 & 6.55 & $-36.72^{*}$ & $-32.05^{*}$ \\
\hline 32 & CLN 2116 B-1 x Arka Meghali & $-23.52^{*}$ & $-27.41^{*}$ & -6.19 & $8.22 *$ & $-18.07 *$ & $-11.41 *$ & $-19.94 *$ & $-18.91^{*}$ \\
\hline 33 & CLN 2123 A-1 x Arka Meghali & $-21.73^{*}$ & $-32.26^{*}$ & $5.05^{*}$ & $8.01 *$ & -3.52 & 3.16 & $-24.73 *$ & $-11.22^{*}$ \\
\hline 34 & BWR-1 x Arka Meghali & $-41.21^{*}$ & $-40.69 *$ & 15.21 & $11.47^{*}$ & 16.55 & 2.55 & $-23.10^{*}$ & $-9.29 *$ \\
\hline 35 & BWR-5 x Arka Meghali & $-26.09 *$ & $-34.22^{*}$ & $-6.51^{*}$ & 5.63 & 22.04 & 6.19 & $-8.42^{*}$ & $7.73^{*}$ \\
\hline 36 & EC-528372 x Arka Meghali & $-20.08 *$ & $-30.84 *$ & $11.28^{*}$ & $11.04 *$ & $-14.37 *$ & $-7.40^{*}$ & $-37.50 *$ & $-26.28^{*}$ \\
\hline 37 & EC-521041 x Arka Meghali & $-20.77^{*}$ & $-32.67 *$ & -1.47 & 1.30 & 0.23 & 7.16 & $5.25^{*}$ & $9.59 *$ \\
\hline 38 & EC-526146 x Arka Meghali & $-31.71^{*}$ & $-31.10^{*}$ & 0.00 & 0.65 & 0.59 & 3.52 & $-28.04 *$ & $-20.19^{*}$ \\
\hline 39 & EC-5863 x Arka Meghali & $-20.73^{*}$ & $-29.46^{*}$ & -14.18 & -3.03 & 3.54 & 6.55 & $-34.93 *$ & $-30.13^{*}$ \\
\hline 40 & EC-521079 x Arka Meghali & -3.17 & $-33.20^{*}$ & 7.53 & $8.22 *$ & $-15.60^{*}$ & $-8.74^{*}$ & $7.48^{*}$ & $-26.28^{*}$ \\
\hline
\end{tabular}


Table.3 Estimation of heterosis for fruits firmness, pericarp thickness, number of locules per fruit and number of fruits per cluster

\begin{tabular}{|c|c|c|c|c|c|c|c|c|c|}
\hline \multicolumn{10}{|c|}{ Percent increase or decrease over } \\
\hline \multirow[t]{2}{*}{ Sr. No } & \multirow[t]{2}{*}{ Crosses } & \multicolumn{2}{|c|}{ Fruit firmness $\left(\mathrm{kg}^{\prime} / \mathrm{cm}^{2}\right)$} & \multicolumn{2}{|c|}{ Pericarp thickness (mm) } & \multicolumn{2}{|c|}{ Number of locules per fruit } & \multicolumn{2}{|c|}{ Number of fruits per cluster } \\
\hline & & BP & SC & BP & SC & BP & SC & BP & SC \\
\hline 1 & CLN 2070 B-1 x FT-5 & $15.11^{*}$ & $39.94 *$ & $-23.23^{*}$ & $-23.71^{*}$ & -8.77 & $-18.17^{*}$ & -9.80 & $-17.11 *$ \\
\hline 2 & CLN 2116 B-1 x FT-5 & 9.48 & $38.85^{*}$ & $-23.89 *$ & $-12.17 *$ & $18.45^{*}$ & -0.96 & -5.46 & -6.30 \\
\hline 3 & CLN 2123 A-1 x FT-5 & $-22.61^{*}$ & $18.29 *$ & $-4.23^{*}$ & $-12.83^{*}$ & $-24.91 *$ & $-13.88^{*}$ & -14.75 & -6.30 \\
\hline 4 & BWR-1 $\times$ FT-5 & $-18.30^{*}$ & -5.32 & $-28.68 *$ & $-37.83^{*}$ & 29.06* & 8.53 & $-29.41 *$ & $-35.14 *$ \\
\hline 5 & BWR-5 x FT-5 & 3.45 & 11.51 & $-21.29 *$ & $-21.79^{*}$ & $-29.00 *$ & $-17.31^{*}$ & 4.00 & -6.30 \\
\hline 6 & EC-528372 x FT-5 & 0.20 & 8.02 & $-34.44 *$ & $-24.37 *$ & $22.11 *$ & 5.94 & -9.09 & -9.92 \\
\hline 7 & EC-521041 x FT-5 & $22.41^{*}$ & $55.24^{*}$ & 9.86 & 0.00 & 12.50 & -12.14 & -14.75 & -6.30 \\
\hline 8 & EC-526146 x FT-5 & $-14.59^{*}$ & 15.23 & $52.54 *$ & $15.38^{*}$ & $-17.77^{*}$ & $-36.25^{*}$ & 11.00 & 0.00 \\
\hline 9 & EC-5863 x FT-5 & $-18.22^{*}$ & $24.63^{*}$ & $-5.66^{*}$ & -3.85 & $-50.10^{*}$ & $-36.25^{*}$ & $-40.50 *$ & $-35.14 *$ \\
\hline 10 & EC-521079 x FT-5 & $-17.92 *$ & -4.89 & $-38.89 *$ & $-29.48 *$ & $45.42 *$ & 6.80 & -10.74 & -2.70 \\
\hline 11 & CLN 2070 B-1 x Solan Lalima & $-15.46^{*}$ & $32.72^{*}$ & $-13.21^{*}$ & $-11.54 *$ & $-44.21 *$ & $-37.98^{*}$ & 0.00 & 9.92 \\
\hline 12 & CLN 2116 B-1 x Solan Lalima & $-10.86^{*}$ & $39.94 *$ & $-5.03^{*}$ & -3.21 & $-15.54 *$ & $-31.09 *$ & $7.44^{*}$ & $17.11^{*}$ \\
\hline 13 & CLN 2123 A-1 x Solan Lalima & -2.51 & $53.06^{*}$ & 15.48 & $14.75^{*}$ & $-51.97 *$ & $-36.25 *$ & 4.51 & 4.51 \\
\hline 14 & BWR-1 x Solan Lalima & $-41.51^{*}$ & -8.17 & $-44.44 *$ & $-35.90^{*}$ & $15.25 *$ & $-22.48^{*}$ & 0.90 & 0.89 \\
\hline 15 & BWR-5 x Solan Lalima & -4.32 & $50.22^{*}$ & $8.45^{*}$ & -1.29 & $-67.64 *$ & $-36.25^{*}$ & $-21.31 *$ & -13.51 \\
\hline 16 & EC-528372 x Solan Lalima & $-27.58^{*}$ & 13.70 & $20.83^{*}$ & -7.06 & 3.27 & $-13.88^{*}$ & -1.80 & -1.81 \\
\hline 17 & EC-521041 x Solan Lalima & -2.65 & $52.84^{*}$ & 18.71 & $17.94 *$ & -3.03 & $-39.72^{*}$ & 1.70 & 8.11 \\
\hline 18 & EC-526146 x Solan Lalima & -6.69 & $46.50^{*}$ & $-26.67 *$ & $-15.38^{*}$ & -8.23 & $-32.82^{*}$ & 3.39 & 9.92 \\
\hline 19 & EC-5863 x Solan Lalima & -0.42 & $56.34 *$ & 30.28 & $18.60^{*}$ & $-41.79 *$ & $-27.65^{*}$ & -2.46 & 7.22 \\
\hline 20 & EC-521079 x Solan Lalima & $-43.45 *$ & -11.22 & $-7.63^{*}$ & $-30.13^{*}$ & $23.50^{*}$ & $-13.88^{*}$ & -0.85 & 5.41 \\
\hline 21 & CLN 2070 B-1 x Solan Vajr & $-30.63^{*}$ & 10.42 & -10.97 & $-11.54^{*}$ & -6.79 & $-15.58^{*}$ & 27.27 & 0.89 \\
\hline 22 & CLN 2116 B-1 x Solan Vajr & $-34.20^{*}$ & 4.74 & $-39.44 *$ & $-30.13^{*}$ & -8.75 & $-25.06^{*}$ & -3.64 & -4.51 \\
\hline 23 & CLN 2123 A-1 x Solan Vajr & -7.97 & $46.50^{*}$ & 14.09 & 3.85 & $-30.09 *$ & $-18.17^{*}$ & 0.00 & 9.92 \\
\hline 24 & BWR-1 x Solan Vajr & $-31.04 *$ & 9.76 & 13.04 & 0.00 & $39.54 *$ & 4.21 & 21.43 & 7.22 \\
\hline 25 & BWR-5 x Solan Vajr & $-41.62^{*}$ & -7.07 & $-28.03^{*}$ & $-27.56^{*}$ & -2.92 & 3.36 & $-31.09 *$ & $-26.14^{*}$ \\
\hline 26 & EC-528372 x Solan Vajr & $-45.05^{*}$ & -12.54 & $-39.44 *$ & $-30.13^{*}$ & $20.47 *$ & 4.21 & $-38.66 *$ & $-34.24 *$ \\
\hline 27 & EC-521041 x Solan Vajr & $-27.75 *$ & 15.01 & $-10.83^{*}$ & $-10.25^{*}$ & $33.44 *$ & -9.56 & -11.48 & -2.70 \\
\hline 28 & EC-526146 x Solan Vajr & $-24.59^{*}$ & $20.04 *$ & $-19.11 *$ & $-18.60^{*}$ & 8.23 & $-22.48^{*}$ & -7.56 & -0.89 \\
\hline 29 & EC-5863 x Solan Vajr & 0.96 & $60.71^{*}$ & $-3.87^{*}$ & -4.48 & -10.82 & -5.25 & $-9.57^{*}$ & -6.30 \\
\hline 30 & EC-521079 x Solan Vajr & $-20.74 *$ & $26.17^{*}$ & -19.44 & -7.06 & -2.59 & $-13.00^{*}$ & $-20.00^{*}$ & $-17.11^{*}$ \\
\hline 31 & CLN 2070 B-1 x Arka Meghali & $-33.27 *$ & $-18.88^{*}$ & $-22.00 *$ & $-25.00^{*}$ & -2.97 & $-13.00^{*}$ & -14.75 & -6.30 \\
\hline 32 & CLN 2116 B-1 x Arka Meghali & $-16.72^{*}$ & 5.61 & -31.33 & $-33.98^{*}$ & 6.63 & -4.39 & $-37.39 *$ & $-35.14 *$ \\
\hline 33 & CLN 2123 A-1 x Arka Meghali & $-12.88^{*}$ & $33.16^{*}$ & $-21.86^{*}$ & -8.33 & -9.51 & -1.81 & $-24.62^{*}$ & -11.70 \\
\hline 34 & BWR-1 x Arka Meghali & $-20.75^{*}$ & -8.16 & -26.23 & $-13.46^{*}$ & 5.57 & -4.39 & $-32.31 *$ & $-20.73^{*}$ \\
\hline 35 & BWR-5 x Arka Meghali & 10.68 & 6.49 & $-47.54 *$ & $-38.46^{*}$ & -6.83 & -0.96 & $-47.69 *$ & $-38.73^{*}$ \\
\hline 36 & EC-528372 x Arka Meghali & $32.44^{*}$ & $18.73^{*}$ & $-38.80^{*}$ & $-28.21^{*}$ & 2.38 & -7.83 & -33.85 & $-22.51^{*}$ \\
\hline 37 & EC-521041 x Arka Meghali & $-36.03^{*}$ & $-18.88^{*}$ & $-36.77^{*}$ & $-37.17^{*}$ & -4.90 & $-14.73^{*}$ & $-33.93 *$ & $-33.32^{*}$ \\
\hline 38 & EC-526146 x Arka Meghali & $-35.17 *$ & -12.54 & $-35.56 *$ & $-25.63^{*}$ & 11.44 & -0.08 & $-28.57 *$ & $-27.92^{*}$ \\
\hline 39 & EC-5863 x Arka Meghali & $-14.63^{*}$ & $30.10^{*}$ & $-16.20^{*}$ & $-23.71^{*}$ & $-16.43^{*}$ & -12.14 & $-27.87 *$ & $-20.73^{*}$ \\
\hline 40 & EC-521079 x Arka Meghali & $-14.34 *$ & -0.73 & $-13.56^{*}$ & $-34.62^{*}$ & $99.92 *$ & 2.51 & $-34.82^{*}$ & $-34.24 *$ \\
\hline
\end{tabular}


Table.4 Estimation of heterosis for number of fruits per plant, average fruit weight, marketable fruit yield per plant and yield

\begin{tabular}{|c|c|c|c|c|c|c|c|c|c|}
\hline \multicolumn{10}{|c|}{ Percent increase or decrease over } \\
\hline \multirow[t]{2}{*}{ Sr. No. } & \multirow[t]{2}{*}{ Crosses } & \multicolumn{2}{|c|}{ Number of fruits per plant } & \multicolumn{2}{|c|}{ Average fruit weight (g) } & \multicolumn{2}{|c|}{ Marketable fruit yield per plant $(\mathrm{g})$} & \multicolumn{2}{|c|}{ Marketable fruit yield (q/ha) } \\
\hline & & BP & $\mathrm{SC}$ & BP & SC & BP & $\mathrm{SC}$ & BP & SC8 \\
\hline 1 & CLN 2070 B-1 x FT-5 & $-25.70^{*}$ & $-26.62 *$ & $40.42 *$ & $-15.32 *$ & $22.33^{*}$ & $-24.46^{*}$ & $22.33^{*}$ & -24.46 \\
\hline 2 & CLN 2116 B-1 x FT-5 & 7.79 & 6.47 & $20.84 *$ & $-28.20^{*}$ & $-4.21^{*}$ & $-43.52^{*}$ & $-4.21 *$ & -43.52 \\
\hline 3 & CLN $2123 \mathrm{~A}-1 \times \mathrm{FT}-5$ & $-26.63 *$ & $-16.42 *$ & $26.92^{*}$ & -2.36 & $-19.61 *$ & $-39.74 *$ & $-19.61 *$ & -39.74 \\
\hline 4 & BWR-1 x FT-5 & -2.78 & -3.98 & $-16.46^{*}$ & $-50.37 *$ & $-8.97 *$ & $-46.32^{*}$ & $-8.97 *$ & -46.32 \\
\hline 5 & BWR-5 x FT-5 & $-20.92^{*}$ & $-21.89 *$ & $24.84^{*}$ & $-25.83 *$ & $-23.02 *$ & $-54.61^{*}$ & $-23.02 *$ & $-54.61 *$ \\
\hline 6 & EC-528372 $\times$ FT-5 & 4.02 & 2.74 & $-13.85 *$ & $-48.82 *$ & $-31.77^{*}$ & -59.77 & $-31.77 *$ & -59.77 \\
\hline 7 & EC-521041 x FT-5 & $-29.20^{*}$ & $-20.40^{*}$ & $37.21^{*}$ & -0.24 & $-30.68^{*}$ & $-48.28 *$ & $-30.68^{*}$ & $-48.28 *$ \\
\hline 8 & EC-526146 x FT-5 & $11.82^{*}$ & 10.45 & $30.31^{*}$ & 8.64* & $16.13^{*}$ & $-23.73^{*}$ & $16.13^{*}$ & $-23.73 *$ \\
\hline 9 & EC-5863 x FT-5 & -8.16 & 0.75 & $-11.63^{*}$ & $-34.39 *$ & $-24.37 *$ & $-43.40^{*}$ & $-24.37 *$ & -43.40 \\
\hline 10 & EC-521079 x FT-5 & $-17.90^{*}$ & $-18.91^{*}$ & $20.43^{*}$ & $-28.44^{*}$ & $-11.68^{*}$ & $-47.92^{*}$ & $-11.68^{*}$ & $-47.92 *$ \\
\hline 11 & CLN 2070 B-1 x Solan Lalima & -8.45 & 7.71 & -4.13 & $-29.91 *$ & -2.28 & $-26.66^{*}$ & -2.28 & -26.66 \\
\hline 12 & CLN 2116 B-1 x Solan Lalima & $-33.61 *$ & $-21.89 *$ & $29.98^{*}$ & $-4.97 *$ & -1.01 & $-25.70^{*}$ & -1.01 & -25.70 \\
\hline 13 & CLN 2123 A-1 x Solan Lalima & -2.53 & $14.68^{*}$ & $7.31^{*}$ & $-17.44 *$ & $67.15^{*}$ & $25.46^{*}$ & $67.15^{*}$ & $25.46^{*}$ \\
\hline 14 & BWR-1 x Solan Lalima & $-32.55 *$ & $-20.65 *$ & $-20.62 *$ & $-41.97 *$ & $-42.96^{*}$ & $-57.18^{*}$ & $-42.96 *$ & -57.18 \\
\hline 15 & BWR-5 x Solan Lalima & -8.45 & 7.71 & $29.21^{*}$ & $-5.54 *$ & -10.268 & $-32.64 *$ & -10.26 & -32.64 \\
\hline 16 & EC-528372 x Solan Lalima & -7.60 & 8.71 & -1.00 & $-27.63^{*}$ & $-9.19^{*}$ & $-31.84 *$ & $-9.19^{*}$ & -31.84 \\
\hline 17 & EC-521041 x Solan Lalima & 3.82 & $22.14^{*}$ & $41.25^{*}$ & 3.26 & $35.80^{*}$ & $1.93^{*}$ & $35.80^{*}$ & $1.93^{*}$ \\
\hline 18 & EC-526146 x Solan Lalima & -1.26 & $16.17^{*}$ & $-23.46^{*}$ & $-36.19 *$ & $64.87 *$ & $23.75^{*}$ & $64.87^{*}$ & $23.75^{*}$ \\
\hline 19 & EC-5863 x Solan Lalima & $10.66^{*}$ & $30.20^{*}$ & $37.84^{*}$ & 2.33 & $68.18^{*}$ & $26.24 *$ & $68.18^{*}$ & $26.24^{*}$ \\
\hline 20 & EC-521079 x Solan Lalima & $-23.88^{*}$ & -10.45 & $-29.43 *$ & $-48.41^{*}$ & $-51.32^{*}$ & $-63.46^{*}$ & $-51.32^{*}$ & $-63.46^{*}$ \\
\hline 21 & CLN 2070 B-1 x Solan Vajr & -5.63 & 4.23 & $-40.94 *$ & $-51.67 *$ & $-39.76^{*}$ & $-55.05^{*}$ & $-39.76^{*}$ & $-55.05 *$ \\
\hline 22 & CLN 2116 B-1 x Solan Vajr & $-9.91 *$ & -0.50 & $-37.25 *$ & $-48.66 *$ & $-49.10^{*}$ & $-62.03^{*}$ & $-49.10^{*}$ & $-62.03 *$ \\
\hline 23 & CLN 2123 A-1 x Solan Vajr & $-41.48 *$ & $-33.33 *$ & $30.99^{*}$ & $7.17^{*}$ & $48.23 *$ & $11.11^{*}$ & $48.23^{*}$ & $11.11^{*}$ \\
\hline 24 & BWR-1 x Solan Vajr & $-13.51^{*}$ & -4.48 & 2.90 & $-15.81^{*}$ & $-16.01 *$ & $-37.34 *$ & $-16.01 *$ & $-37.34 *$ \\
\hline 25 & BWR-5 x Solan Vajr & $-18.92 *$ & -10.45 & $-36.46^{*}$ & $-48.00^{*}$ & $-58.52^{*}$ & $-69.06^{*}$ & $-58.52 *$ & -69.06 \\
\hline 26 & EC-528372 x Solan Vajr & $-24.77 *$ & $-16.91 *$ & $14.94 *$ & $-5.95^{*}$ & -1.34 & $-26.39^{*}$ & -1.34 & $-26.39^{*}$ \\
\hline 27 & EC-521041 x Solan Vajr & $-21.65^{*}$ & $-11.94 *$ & $7.68^{*}$ & $-11.90^{*}$ & -0.17 & $-25.51^{*}$ & -0.17 & $-25.51^{*}$ \\
\hline 28 & EC-526146 x Solan Vajr & $-32.66 *$ & $-25.62 *$ & $-28.05 *$ & $-40.02 *$ & $-44.64 *$ & $-58.70^{*}$ & $-44.64 *$ & -58.70 \\
\hline 29 & EC-5863 x Solan Vajr & $11.32^{*}$ & $22.50^{*}$ & $20.02^{*}$ & -1.79 & $68.00 *$ & $25.73^{*}$ & $68.00^{*}$ & $25.73 *$ \\
\hline 30 & EC-521079 x Solan Vajr & $-23.65 *$ & $-15.67 *$ & $-7.77^{*}$ & $-24.53 *$ & $-21.33^{*}$ & $-41.31 *$ & $-21.33^{*}$ & $-41.31^{*}$ \\
\hline 31 & CLN 2070 B-1 x Arka Meghali & $-22.22 *$ & $-32.09 *$ & -4.32 & $-42.30 *$ & $-53.82 *$ & $-71.48 *$ & $-53.82 *$ & -71.48 \\
\hline 32 & CLN 2116 B-1 x Arka Meghali & -5.66 & $-25.62 *$ & -5.72 & $-44.83 *$ & $-47.34 *$ & $-72.71 *$ & $-47.34 *$ & $-72.71 *$ \\
\hline 33 & CLN 2123 A-1 x Arka Meghali & $-27.72 *$ & $-17.66 *$ & $-20.75 *$ & $-39.04 *$ & $-55.36^{*}$ & $-66.54 *$ & $-55.36 *$ & -66.54 \\
\hline 34 & BWR-1 x Arka Meghali & $-15.75^{*}$ & $-28.11 *$ & -7.74 & $-52.49 *$ & $-39.95^{*}$ & $-74.30^{*}$ & $-39.95^{*}$ & $-74.30^{*}$ \\
\hline 35 & BWR-5 x Arka Meghali & 3.98 & $-28.61 *$ & $67.58^{*}$ & $-13.69 *$ & $46.65^{*}$ & $-40.41^{*}$ & $46.65^{*}$ & $-40.41 *$ \\
\hline 36 & EC-528372 x Arka Meghali & $-20.42^{*}$ & $-36.07 *$ & $35.46^{*}$ & $-30.24 *$ & $-28.41^{*}$ & $-70.91 *$ & $-28.41^{*}$ & $-70.91 *$ \\
\hline 37 & EC-521041 x Arka Meghali & $-27.21 *$ & $-18.16^{*}$ & $-9.65^{*}$ & $-34.31 *$ & -18.79 & $-39.41^{*}$ & -18.79 & $-39.41 *$ \\
\hline 38 & EC-526146 x Arka Meghali & $-29.26^{*}$ & $-30.85^{*}$ & $-35.09 *$ & $-45.88 *$ & $-55.88^{*}$ & $-71.02 *$ & $-55.88^{*}$ & -71.02 \\
\hline 39 & EC-5863 x Arka Meghali & $-33.11 *$ & $-26.62^{*}$ & -0.87 & $-26.41 *$ & $-63.52 *$ & $-72.70^{*}$ & $-63.52 *$ & -72.70 \\
\hline 40 & EC-521079 x Arka Meghali & -7.13 & $-31.84 *$ & -3.63 & $-50.37 *$ & $-40.89^{*}$ & $-75.98 *$ & $-40.89 *$ & -75.98 \\
\hline
\end{tabular}


Table.5 Estimation of heterosis for alternaria blight severity, buckeye rot incidence, total soluble solids, ascorbic acid content and lycopene content

\begin{tabular}{|c|c|c|c|c|c|c|c|c|c|c|c|}
\hline \multirow[t]{2}{*}{ Sr. No } & \multirow[t]{2}{*}{ Crosses } & \multicolumn{2}{|c|}{ Alternaria blight severity $(\%)$} & \multicolumn{2}{|c|}{ Buckeye rot incidence (\%) } & \multicolumn{2}{|c|}{ Total soluble solids ( ${ }^{\circ} \mathrm{Brix}$ ) } & \multicolumn{2}{|c|}{ Ascorbic acid content $(\mathrm{mg} / \mathbf{1 0 0 g})$} & \multicolumn{2}{|c|}{ Lycopene content (mg/100g) } \\
\hline & & BP & SC & BP & SC & BP & SC & BP & SC & BP & $\mathrm{SC}$ \\
\hline 1 & CLN 2070 B-1 x FT-5 & -9.61 & -3.83 & $22.07 *$ & $26.05^{*}$ & $11.50^{*}$ & 5.00 & $-8.09 *$ & 3.56 & 0.77 & $-14.06^{*}$ \\
\hline 2 & CLN 2116 B-1 x FT-5 & -10.98 & -5.29 & -4.47 & -0.72 & -19.38 & $-13.33^{*}$ & $-22.67 *$ & $-12.44 *$ & $-34.79^{*}$ & $-33.46^{*}$ \\
\hline 3 & CLN 2123 A-1 x FT-5 & $-24.70^{*}$ & $-19.89^{*}$ & -16.30 & -13.18 & $-16.81^{*}$ & $-21.68 *$ & -0.40 & $11.11^{*}$ & $5.29^{*}$ & $14.65 *$ \\
\hline 4 & BWR-1 x FT-5 & $-35.21 *$ & -9.67 & $-18.58^{*}$ & 9.89 & 5.31 & -0.82 & $-6.34 *$ & -3.22 & 23.64 & $-17.29 *$ \\
\hline 5 & BWR-5 x FT-5 & 13.17 & 8.21 & 12.12 & 9.06 & -7.34 & $-15.83^{*}$ & 8.60 & 7.33 & $-40.34 *$ & $-49.11^{*}$ \\
\hline 6 & EC-528372 x FT-5 & $0.58^{*}$ & -5.29 & $23.30^{*}$ & 13.70 & $-45.74^{*}$ & $-41.68^{*}$ & $-13.91 *$ & $-10.22^{*}$ & $-29.79^{*}$ & $-28.34 *$ \\
\hline 7 & EC-521041 x FT-5 & 10.76 & 8.94 & 8.92 & 1.03 & 0.00 & -9.18 & 11.42 & $14.33^{*}$ & $0.74 *$ & 9.71 \\
\hline 8 & EC-526146 x FT-5 & -39.14 & $-15.15^{*}$ & -22.54 & 4.63 & $8.26^{*}$ & -1.68 & $10.65^{*}$ & 4.44 & $38.78^{*}$ & -7.13 \\
\hline 9 & EC-5863 x FT-5 & 2.48 & -2.01 & $35.71^{*}$ & $31.00^{*}$ & -7.08 & $-12.50^{*}$ & 3.34 & $10.00^{*}$ & $12.24 *$ & 6.93 \\
\hline 10 & EC-521079 x FT-5 & 32.28 & $14.42^{*}$ & $75.04 *$ & 10.09 & -15.50 & -9.18 & $-14.90^{*}$ & -4.78 & -26.29 & $-24.78^{*}$ \\
\hline 11 & CLN 2070 B-1 x Solan Lalima & $-32.28 *$ & $-33.39^{*}$ & $-17.63^{*}$ & $-38.00 *$ & 4.43 & -1.68 & $-13.11^{*}$ & -4.22 & $-18.68 *$ & $-11.44^{*}$ \\
\hline 12 & CLN 2116 B-1 x Solan Lalima & $-62.17 *$ & $-47.26^{*}$ & $-52.40^{*}$ & $-35.84 *$ & 22.12 & $15.00^{*}$ & $-1.42^{*}$ & 0.56 & $-8.49 *$ & $-12.81^{*}$ \\
\hline 13 & CLN 2123 A-1 x Solan Lalima & -27.01 & $-13.69^{*}$ & -50.99 & $-37.59 *$ & -0.82 & 0.83 & 7.83 & 7.11 & $39.92^{*}$ & $19.33^{*}$ \\
\hline 14 & BWR-1 x Solan Lalima & $-54.48 *$ & $-46.17^{*}$ & $-48.28^{*}$ & $-35.22 *$ & $-16.28^{*}$ & $-10.00^{*}$ & $-9.86^{*}$ & -5.56 & $-13.64 *$ & $-11.87^{*}$ \\
\hline 15 & BWR-5 x Solan Lalima & -23.30 & -9.31 & $-46.72^{*}$ & $-32.55 *$ & $-8.20^{*}$ & -6.68 & $-9.91 *$ & -7.11 & $-23.56^{*}$ & $-16.75^{*}$ \\
\hline 16 & EC-528372 x Solan Lalima & -28.40 & -0.18 & $-39.45^{*}$ & $-17.51^{*}$ & $-19.67 *$ & $-18.33^{*}$ & $-12.18^{*}$ & $-16.67 *$ & $6.52 *$ & $-28.71^{*}$ \\
\hline 17 & EC-521041 x Solan Lalima & $-43.32 *$ & $-45.80^{*}$ & $-47.08^{*}$ & $-48.51^{*}$ & 17.65 & 0.00 & 0.32 & 6.22 & $52.05^{*}$ & $29.67 *$ \\
\hline 18 & EC-526146 x Solan Lalima & $-43.05 *$ & $-46.90^{*}$ & -24.40 & $-29.56^{*}$ & $-13.18^{*}$ & -6.68 & 3.19 & $14.89^{*}$ & $-12.07 *$ & -10.28 \\
\hline 19 & EC-5863 x Solan Lalima & -33.40 & $-34.49^{*}$ & $-44.73^{*}$ & $-47.79 *$ & $2.83^{*}$ & -9.18 & -2.23 & 7.22 & $11.85^{*}$ & $21.80^{*}$ \\
\hline 20 & EC-521079 x Solan Lalima & $9.03 *$ & $52.01^{*}$ & $-24.60^{*}$ & 3.40 & $4.90^{*}$ & $-10.83^{*}$ & $-4.82 *$ & -3.44 & $4.88^{*}$ & $-18.59^{*}$ \\
\hline 21 & CLN 2070 B-1 x Solan Vajr & $-51.94 *$ & $-45.80^{*}$ & $-57.42^{*}$ & $-42.64 *$ & $-6.93^{*}$ & $-21.68 *$ & 3.66 & 2.22 & $-17.61^{*}$ & $-29.74 *$ \\
\hline 22 & CLN 2116 B-1 x Solan Vajr & $1.29^{*}$ & $14.23 *$ & $-17.81^{*}$ & 10.92 & -12.40 & -5.83 & $-7.74 *$ & -4.00 & 4.64 & 6.78 \\
\hline 23 & CLN 2123 A-1 x Solan Vajr & $-51.78^{*}$ & $-45.62^{*}$ & -45.34 & $-26.78^{*}$ & 2.83 & -9.18 & 2.12 & 4.56 & -7.36 & 0.87 \\
\hline 24 & BWR-1 x Solan Vajr & $-43.72^{*}$ & $-21.53^{*}$ & $-23.99^{*}$ & 2.47 & 16.83 & -1.68 & $1.48^{*}$ & -4.44 & $28.65^{*}$ & $-13.91 *$ \\
\hline 25 & BWR-5 x Solan Vajr & 5.34 & 0.73 & $7.62^{*}$ & 10.61 & $11.22^{*}$ & -0.82 & -10.73 & -1.11 & $-30.76^{*}$ & $-34.55^{*}$ \\
\hline 26 & EC-528372 x Solan Vajr & $-38.95^{*}$ & $-42.52^{*}$ & $-27.35^{*}$ & $-25.54 *$ & -26.36 & $-20.83^{*}$ & $-21.22^{*}$ & -8.44 & $-38.93 *$ & $-37.68 *$ \\
\hline 27 & EC-521041 x Solan Vajr & $-31.54 *$ & $-32.66^{*}$ & $-22.24 *$ & $-20.19 *$ & 9.35 & -2.50 & -0.10 & $14.44 *$ & $-16.20^{*}$ & -8.75 \\
\hline 28 & EC-526146 x Solan Vajr & -30.12 & -2.55 & $-26.05^{*}$ & 0.20 & 17.76 & 5.00 & $-0.73^{*}$ & 5.56 & $-5.32^{*}$ & -10.50 \\
\hline 29 & EC-5863 x Solan Vajr & $-44.28 *$ & $-46.71 *$ & -30.71 & $-32.96 *$ & -23.88 & $-15.00 *$ & $8.11 *$ & $15.56^{*}$ & $8.37 *$ & $19.83^{*}$ \\
\hline 30 & EC-521079 x Solan Vajr & 49.76 & $14.78^{*}$ & $57.80^{*}$ & -0.41 & $-14.93^{*}$ & -5.00 & -14.94 & -4.44 & $-41.00^{*}$ & $-34.77^{*}$ \\
\hline 31 & CLN 2070 B-1 x Arka Meghali & 41.37 & $39.05^{*}$ & 92.84 & $44.59 *$ & -8.96 & 1.68 & $-21.19^{*}$ & $-12.78^{*}$ & -42.98 & $-36.96^{*}$ \\
\hline 32 & CLN 2116 B-1 x Arka Meghali & $-29.06 *$ & -1.09 & $-11.35^{*}$ & $19.57^{*}$ & $-29.10^{*}$ & $-20.83 *$ & -1.74 & 0.67 & -41.20 & $-34.99 *$ \\
\hline 33 & CLN 2123 A-1 x Arka Meghali & 37.02 & $31.02 *$ & 38.90 & $35.01^{*}$ & 0.00 & -5.83 & -14.66 & -5.56 & $-25.44^{*}$ & $-31.40^{*}$ \\
\hline 34 & BWR-1 x Arka Meghali & $45.71 *$ & 11.68 & 92.15 & $30.79 *$ & -5.43 & 1.68 & $-15.69^{*}$ & -2.11 & -38.21 & $-36.96^{*}$ \\
\hline 35 & BWR-5 x Arka Meghali & 37.66 & $35.40^{*}$ & 84.85 & $38.62 *$ & $15.04 *$ & 8.33 & $-26.60^{*}$ & $-16.00^{*}$ & $-43.44 *$ & $-38.40^{*}$ \\
\hline 36 & EC-528372 x Arka Meghali & $-14.92^{*}$ & $18.61^{*}$ & 2.36 & $38.21^{*}$ & $6.20^{*}$ & 0.00 & $-9.52^{*}$ & -3.89 & -33.68 & $-38.99 *$ \\
\hline 37 & EC-521041 x Arka Meghali & 21.71 & $35.04 *$ & -12.24 & $30.48 *$ & $-32.84 *$ & $-25.00^{*}$ & $4.01 *$ & 0.89 & $-10.94 *$ & $-24.05 *$ \\
\hline 38 & EC-526146 x Arka Meghali & 27.30 & $41.24 *$ & -6.89 & $38.41^{*}$ & $-11.94 *$ & -1.68 & $0.87 *$ & 3.33 & $-31.07 *$ & $-29.65^{*}$ \\
\hline 39 & EC-5863 x Arka Meghali & $-10.36^{*}$ & -0.55 & -14.88 & $25.85^{*}$ & -13.43 & -3.33 & -1.54 & -0.78 & $-32.40^{*}$ & $-26.37^{*}$ \\
\hline 40 & EC-521079 x Arka Meghali & $11.13^{*}$ & $54.93 *$ & 1.95 & $51.49 *$ & $-29.85^{*}$ & $-21.68^{*}$ & $-2.04 *$ & -9.33 & 2.51 & $-31.40^{*}$ \\
\hline
\end{tabular}


Desirably significant negative heterosis over better parent ranged from maximum -57.42 per cent (CLN 2070 B-1 x Solan Vajr) to undesirably high incidence of $75.04 \%$ in EC521079 x FT-5 for buckeye rot incidence, respectively over the better parents. Over the standard check, while 14 cross combinations showed significantly lesser disease menace, with EC-521041 x Solan Lalima (-48.51) showing the maximum negative heterosis. For TSS 7 combinations produced significant positive heterosis over the better parent, maximum being in BWR-5 x Arka Meghali $(15.04 \%)$ (Table 5), on the contrary, only one combination (CLN 2116 x Solan Lalima $(15.00 \%)$ developed and evaluated significantly surpassed in positive heterotic effect over the standard check. Gul et al., (2013) reported similar results over better parent. An insight into the (Table 5) revealed that heterosis over better parent was found significant positive in EC-526146 x FT-5 $(10.65 \%)$ followed by 4 more crosses for ascorbic acid content whereas, 6 cross combinations showed significant positive heterosis over the standard check, maximum being in EC-5863 x Solan Vajr (15.56 \%) the results are in line with Anita et al., (2005). Eleven of the cross combinations surpassed the better parent in heterotic values in case of lycopene content maximum being in EC$521041 \times$ Solan Lalima (52.05 \%). Five cross combinations exceeded the standard check in heterotic values as presented in Table 5 maximum was reported in EC-521041 $x$ Solan Lalima (29.67) Mondal et al., (2009) reported similar results over the better parent.

\section{References}

Allard RW 1960. Principles of Plant Breeding. John Wiley and Sons Inc. New York, pp 270-71.

Anita S, Gautam JPS, Upadhyay M and Joshi A 2005. Heterosis for yield and quality characters in tomato. Crop Research
Hissar 29 (2): 285-287.

Baishya KC, Syamal MM and Singh KP 2001. Heterotic studies in tomato (Lycopersicon esculentum Mill.). Vegetable Science 28 (2): 168-169.

Fageria MS, Kohli UK and Dhaka RS 2001. Studies on heterobeltiosis for fruit yield and yield attributing traits in tomato (Lycopersicon esculentum Mill.). Haryana Journal of Horticultural Sciences 30 (1-2): 131-133.

Gaikwad AK and Cheema DS 2010. Studies on heterosis using heat tolerant lines in tomato. Journal of Research Punjab Agriculture University 47 (1-2): 53-57.

Gul R, Rahman H, Tahir M, Naeem M and Ghafoor A 2013. Estimates of heterosis for morphological and flavour attributes in tomato. International Journal of Vegetable Science 19: 256-262.

Joshi A, Vikram A and Thakur KS 2004 Genetics of yield and horticultural traits in tomato (Lycopersicon esculentum Mill.). Vegetable Science 31 (2):112117.

Kumar R, Srivastava K, Somappa J, Kumar S and Singh RK 2012. Heterosis for yield and yield components in tomato (Solanum lycopersicum L.). Electronic Journal of Plant Breeding 3 (2): 800805.

Kurian A, Peter KV and Rajan S 2001. Heterosis for yield components and fruit characters in tomato (Solanum lycopersicum L.). Journal of Tropical Agriculture 39: 5-8.

Mondal C, Sarkar S and Hazra P 2009. Line x Tester analysis of combining ability in tomato (Solanum lycopersicum L.). Journal of Crop and Weed. 5 (1): 53-57.

Panchal BB, Patel NB, Patel AI, Tank RV and Chawda SK 2017. Genetic studies for productivity and its related traits in tomato (Solanum lycopersicum L.) International Journal of Chemical Studies 5 (6): 2116-2121. 
Premalakshme V, Thangaraj T, Veeraragavathatham D and Arumugam T 2006. Heterosis and combining ability in tomato (Solanum lycopersicum L.). Vegetable Science, 32 (1): 47-50

Rao ES, Munshi AD, Singh B and Kumar R 2007. Studies on heterosis and combining ability for yield and resistance to early blight in tomato. Indian Journal of Horticulture 64 (3): 331-334.

Sahu M, Sahu KK, Tirkey A, Upadayay D and Mehta N 2016. Heterosis and inbreeding depression for agromorphological characters in tomato (Lycopersicun esculentum Mill.). International Journal of Farm Science 6 (1): 51-64.
Sharma D and Thakur MC 2007. Evaluation of diallel progenies for yield and its contributing traits in tomato under midhill conditions. Indian Journal of Horticulture 65 (3): 297-301.

Singh CB, Rai N, Singh RK, Singh MC, Singh AK and Chaturvedi AK 2008. Heterosis combining ability and gene action studies in tomato (Solanum lycopersicum L.). Vegetable Science 35 (2): 132-135.

Yadav SK, Singh BK, Baranwal DK and Solankey SS 2013. Genetic study of heterosis for yield and quality components in tomato (Solanum lycopersicum L.). African Journal of Agriculture Research 8 (44): 55855591.

\section{How to cite this article:}

Shilpi Khar and Divya Arti. 2019. Heterosis Studies for Yield and Yield Attributing Traits in Tomato (Solanum lycopersicum L.) under North Western Himalayan Region, India. Int.J.Curr.Microbiol.App.Sci. 8(01): 52-61. doi: https://doi.org/10.20546/ijcmas.2019.801.007 